\title{
Efficacy of Secukinumab on Moderate-to-severe Plaque Psoriasis Affecting Different Body Regions: a Pooled Analysis of Four Phase 3 Studies
}

\author{
Alan Menter (D) Jennifer C. Cather · Michael Jarratt · Xiangyi Meng • \\ Adriana Guana · Judit Nyirady
}

Received: May 26, 2016 / Published online: August 30, 2016

(C) The Author(s) 2016. This article is published with open access at Springerlink.com

\begin{abstract}
Introduction: The impact of psoriasis varies with the body region affected. In addition, patients have different perceptions of disease improvement and treatment satisfaction based on the location of skin clearance with treatment. The monoclonal antibody secukinumab selectively targets interleukin-17A-a central cytokine of psoriasis-and provides rapid and sustained clearance for moderate-to-severe psoriasis affecting all body regions. The objective of this study was to evaluate the efficacy of
\end{abstract}

Enhanced content To view enhanced content for this article go to http://www.medengine.com/Redeem/ 9885F0606121AB27.

A. Menter $(\bowtie)$

Baylor University Medical Center, Dallas, TX, USA

e-mail: amderm@gmail.com

J. C. Cather

Modern Research Associates and Modern

Dermatology, A Baylor Health Texas Affiliate,

Dallas, TX, USA

M. Jarratt

DermResearch Inc., Austin, TX, USA

X. Meng · A. Guana · J. Nyirady

Novartis Pharmaceuticals Corporation,

East Hanover, NJ, USA secukinumab on moderate-to-severe psoriasis affecting the trunk, upper limbs, and lower limbs.

Methods: Data were pooled from four phase 3 studies. To be included in the analysis for each body region, patients were required to have a Psoriasis Area and Severity Index (PASI) score $\geq 12$ for that body region and psoriasis covering $\geq 10 \%$ of the surface area of that region. Secukinumab was administered at Baseline, Weeks 1, 2 and 3, and then every 4 weeks from Week 4 to 48 .

Results: Across the trunk, upper limbs, and lower limbs, initial PASI subscore responses were sustained to Week 52. At Week 52, trunk (T) PASI $90 \backslash 100$ responses were achieved by $78.4 \% \backslash 71.1 \%$ of patients receiving secukinumab $300 \mathrm{mg}$, respectively, and by $66.3 \% \backslash 56.9 \%$ of patients receiving secukinumab $150 \mathrm{mg}$, respectively. At Week 52, upper limb (UL) PASI $90 \backslash 100$ responses were achieved by $67.3 \% \backslash 59.1 \%$ of patients receiving secukinumab $300 \mathrm{mg}$, respectively, and by $50.3 \% \backslash 43.3 \%$ of patients receiving secukinumab $150 \mathrm{mg}$, respectively. At Week 52, lower limb (LL) PASI 90\100 responses were achieved by $63.9 \% \backslash 55.3 \%$ of patients receiving secukinumab $300 \mathrm{mg}$, respectively, 
and by $45.1 \% \backslash 36.4 \%$ of patients receiving secukinumab $150 \mathrm{mg}$, respectively. A 50\% reduction in mean PASI subscore occurred after 2.8, 2.9, and 3.4 weeks with secukinumab $300 \mathrm{mg}$ on the trunk, upper limbs, and lower limbs, respectively.

Conclusion: Secukinumab provided robust and sustained efficacy for moderate-to-severe psoriasis affecting the trunk, upper limbs, and lower limbs.

Funding: Novartis Pharmaceuticals Corporation.

Trial registration: ClinicalTrials.gov identifiers: NCT01365455, NCT01358578, NCT01555125, and NCT01636687.

Keywords: Lower limbs; Moderate-to-severe psoriasis; Phase 3; Plaque psoriasis; Pooled analysis; Secukinumab; Trunk; Upper limbs

\section{INTRODUCTION}

Secukinumab, a human monoclonal antibody that selectively binds to and neutralizes interleukin-17A, is approved for the treatment of moderate-to-severe plaque psoriasis in the United States and Europe. In phase 3 studies, the efficacy of secukinumab has been demonstrated based on significant improvements in Psoriasis Area and Severity Index (PASI) responses compared with placebo, etanercept, and ustekinumab [1-4].

Although PASI is infrequently used in clinical practice, an improvement from Baseline of at least $75 \%$ (PASI 75) is well established in clinical trials as a clinically meaningful reduction in disease severity that also correlates with improvements in patients' quality of life [5]. As PASI includes separate assessments for psoriasis signs (erythema, thickening, and scaling) on the head and neck, trunk (including the genitals), upper limbs, and lower limbs [5], PASI results can be adequately used to assess the effects of treatment on specific body regions. It is important to evaluate improvements in psoriasis on different body regions as psoriasis, on certain areas, disproportionately impacts quality of life [6, 7]. Accordingly, patients' perceived improvement and satisfaction with treatment may be differently impacted based on the areas where improvement occurs.

In this issue of Dermatology and Therapy, Kircik et al. reported the robust and sustained clearance of psoriatic lesions on the head and neck following treatment with secukinumab $300 \mathrm{mg}$ and secukinumab $150 \mathrm{mg}$ in a pooled population from four phase 3 trials [8]. Here we present the efficacy of secukinumab (300 or $150 \mathrm{mg}$ ), on the trunk, upper limbs, and lower limbs from the same pooled population, using PASI 90 and PASI 100 response rates. These levels of improvement have become the new benchmarks for success in treating patients with moderate-to-severe psoriasis, especially with new biologic agents [9].

\section{METHODS}

\section{Study Design}

Pooled data to Week 52 from four phase 3, randomized, placebo-controlled, double-blind studies [Efficacy of Response and Safety of Two Fixed Secukinumab Regimens in Psoriasis (ERASURE, ClinicalTrials.gov identifier: NCT01365455), Full Year Investigative Examination of Secukinumab vs. Etanercept Using Two Dosing Regimens to Determine Efficacy in Psoriasis (FIXTURE, ClinicalTrials.gov identifier: NCT01358578), First Study of Secukinumab in Pre-filled Syringes in Subjects With Chronic Plaque-type Psoriasis (FEATURE, 
ClinicalTrials.gov identifier: NCT01555125), and Judging the Efficacy of Secukinumab in Patients With Psoriasis Using Autoinjector: A Clinical Trial Evaluation Treatment Results (JUNCTURE, ClinicalTrials.gov identifier: NCT01636687)] of secukinumab in patients with moderate-to-severe plaque psoriasis were used for this analysis. A detailed description of the design for these trials can be found elsewhere [1-3]. Briefly, subjects received secukinumab (300 or $150 \mathrm{mg}$ ) at Baseline, Weeks 1, 2 and 3, and then every 4 weeks from Week 4 to 48 . At Week 12 , patients receiving placebo were randomized to secukinumab.

\section{Study Population}

All studies included adults 18 years of age or older with a diagnosis of moderate-to-severe plaque psoriasis of at least 6 months prior to randomization that was poorly controlled with topical, phototherapy, and $\backslash$ or systemic therapy. To meet the definition of moderate-to-severe psoriasis, patients were required to have a composite PASI score $\geq 12$, an Investigator's Global Assessment modified 2011 (IGA mod 2011) [10] score $\geq 3$, and total affected body surface area $\geq 10 \%$ at Baseline. To be included in the subanalysis for a given body region, patients were required to have a PASI score $\geq 12$ for that specific body region and psoriasis covering $\geq 10 \%$ of the surface area of that region. Exclusion criteria included active, ongoing inflammatory diseases; active, ongoing, chronic or recurrent infectious disease, or evidence of tuberculosis infection; or an underlying condition significantly immunocompromising the patient and $\backslash$ or placing the patient at unacceptable risk for receiving an immunomodulatory therapy (e.g., lymphoproliferative disease, malignancy, history of malignancy within the past 5 years; and $\backslash$ or past medical history of human immunodeficiency virus, hepatitis B, or hepatitis C).

\section{Efficacy Assessments}

In all 4 trials, the coprimary efficacy variables were PASI 75 response rates and IGA mod 2011 $0 \backslash 1$ response rates at Week 12 . The proportion of patients achieving PASI 90 at Week 12 was a key secondary endpoint in all trials.

\section{Statistical Analysis}

Efficacy data from the ERASURE, FIXTURE, FEATURE, and JUNCTURE trials were pooled through Week 52. Missing values were imputed by multiple imputation (MI). The SAS (version 9.3, SAS Institute, Cary, NC, USA) MI procedure was used to generate data sets for 500 imputations. The MI method was used in this study because it more accurately reflects real world response rates than the non-responder imputation method, which underestimates PASI responses [11]. This article is based on previously conducted studies, and does not involve any new studies of human or animal subjects performed by any of the authors.

\section{RESULTS}

\section{Patient Demographics}

Of the 2403 randomized patients, the number eligible for the evaluation of each body region (PASI score $\geq 12$ for that specific body region and psoriasis covering $\geq 10 \%$ of the surface area of that region) who received secukinumab were 1092 for the trunk, 1233 for the upper limbs, and 1309 for the lower limbs. As reported by 
Kircik et al. [8], demographic characteristics for the pooled population were well balanced across secukinumab treatment groups [8]. Briefly, approximately $70 \%$ of patients were male and White and mean PASI scores at randomization were approximately 23 .

\section{Efficacy}

At Week 12 and Week 52, PASI 75 subscore responses on the trunk, upper limbs, and lower limbs were high for patients receiving both secukinumab $300 \mathrm{mg}$ and secukinumab $150 \mathrm{mg}$. PASI 75 subscore response rates at Week 12 were well maintained up to Week 52. The most robust efficacy was observed on the trunk, followed by the upper limbs and then the lower limbs (Table 1).

PASI 90 and PASI 100 subscore response rates to Week 52 for each body region are shown in Figs. 1 and 2, respectively. For all body regions, initial PASI 90 and PASI 100 subscore responses were maintained to Week 52. At Week 16, a numerically greater proportion of patients achieved skin clearance on the trunk than on the upper limbs or the lower limbs. Trunk (T) PASI 90 and TPASI 100 responses were achieved by $82.5 \%$ and $70.0 \%$ of patients receiving secukinumab $300 \mathrm{mg}$, respectively, and by $70.8 \%$ and $58.9 \%$ of patients receiving secukinumab $150 \mathrm{mg}$, respectively. Upper limb (UL) PASI 90 and ULPASI 100 responses were achieved by $72.1 \%$ and $61.4 \%$ of patients receiving secukinumab $300 \mathrm{mg}$, respectively, and by $57.6 \%$ and $43.7 \%$ of patients receiving secukinumab $150 \mathrm{mg}$, respectively. Lower limb (LL) PASI 90 and LLPASI 100 responses were achieved by $68.9 \%$ and $52.9 \%$ of patients receiving secukinumab $300 \mathrm{mg}$, respectively, and by $50.5 \%$ and $35.8 \%$ of patients receiving secukinumab $150 \mathrm{mg}$, respectively.

The trend for a greater response to secukinumab on the trunk than on the upper limbs or the lower limbs was maintained up to Week 52. At Week 52, TPASI 90 and TPASI 100 responses were achieved by $78.4 \%$ and $71.1 \%$ of patients receiving secukinumab $300 \mathrm{mg}$, respectively, and by $66.3 \%$ and $56.9 \%$ of patients receiving secukinumab $150 \mathrm{mg}$, respectively. ULPASI 90 and ULPASI 100

Table 1 Percentage of patients who achieved PASI 75 at Week 12 and Week 52 by body region

\begin{tabular}{lll}
\hline Parameter & Secukinumab 300 mg & Secukinumab 150 mg \\
\hline Week 12 & & \\
TPASI 75 & $88.8 \%(488 / 550)$ & $78.0 \%(423 / 542)$ \\
ULPASI 75 & $83.5 \%(510 / 611)$ & $72.9 \%(454 / 622)$ \\
LLPASI 75 & $81.2 \%(530 / 652)$ & $66.1 \%(434 / 657)$ \\
Week 52 & & \\
TPASI 75 & $90.9 \%(500 / 550)$ & $82.1 \%(445 / 542)$ \\
ULPASI 75 & $85.9 \%(525 / 611)$ & $71.6 \%(445 / 622)$ \\
LLPASI 75 & $82.1 \%(535 / 652)$ & $66.3 \%(436 / 657)$ \\
\hline
\end{tabular}

Missing values were imputed by multiple imputation

LLPASI lower limb Psoriasis Area and Severity Index, TPASI trunk Psoriasis Area and Severity Index, ULPASI upper limb Psoriasis Area and Severity Index 

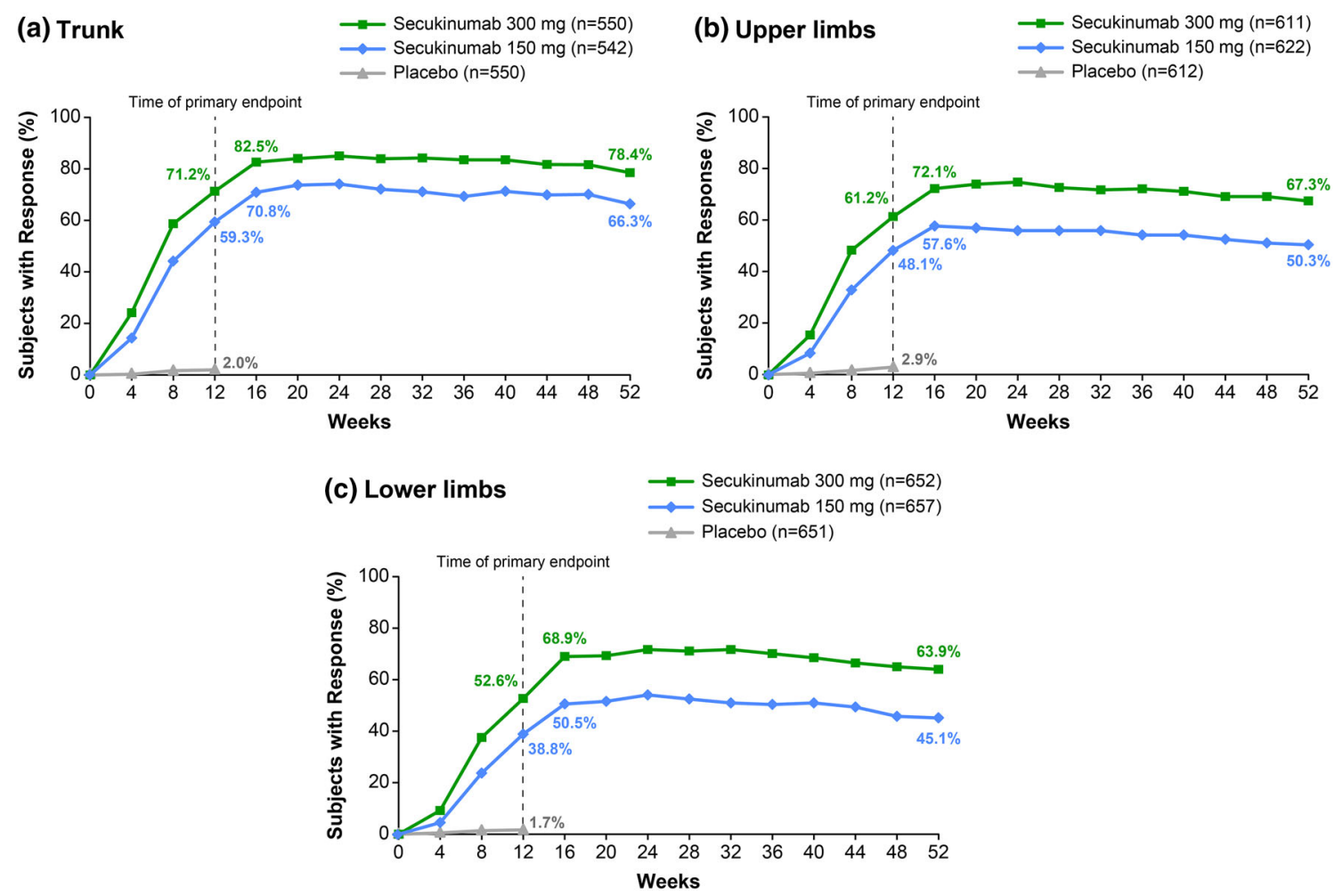

Fig. 1 PASI 90 subscore response rates by body region. Missing values were imputed by multiple imputation. Clinical response rates for a trunk, b upper limbs, and

responses were achieved by $67.3 \%$ and $59.1 \%$ of patients receiving secukinumab $300 \mathrm{mg}$, respectively, and by $50.3 \%$ and $43.3 \%$ of patients receiving secukinumab $150 \mathrm{mg}$, respectively. LLPASI 90 and LLPASI 100 responses were achieved by $63.9 \%$ and $55.3 \%$ of patients receiving secukinumab $300 \mathrm{mg}$, respectively, and by $45.1 \%$ and $36.4 \%$ of patients receiving secukinumab $150 \mathrm{mg}$, respectively.

As shown in Fig. 3, treatment with secukinumab rapidly improved plaque psoriasis, with a $50 \%$ reduction in mean PASI subscore with secukinumab $300 \mathrm{mg}$ observed after 2.8 weeks [95\% confidence interval (CI) 2.6-2.9] on the trunk, 2.9 weeks (95\% CI 2.8-3.1) on the upper limbs, and 3.4 weeks (95\% CI 3.2-3.6) on the lower limbs. With c lower limbs from Baseline to Week 52. $n$ Represents the number of evaluable subjects. PASI $9090 \%$ improvement from Baseline Psoriasis Area and Severity Index

administration of secukinumab $150 \mathrm{mg}$, a 50\% reduction in mean PASI subscore was observed after 3.4 weeks (95\% CI 3.2-3.6) on the trunk, 3.7 weeks (95\% CI 3.5-3.9) on the upper limbs, and 4.5 weeks (95\% CI 4.1-4.9) on the lower limbs.

\section{DISCUSSION}

Results of this pooled analysis show that secukinumab provides rapid and sustained efficacy over 52 weeks in treating patients with moderate-to-severe plaque psoriasis on the trunk, upper limbs, and lower limbs. These findings are consistent with the efficacy of secukinumab observed for psoriasis lesions affecting the head and neck [8], indicating that secukinumab is effective for treating psoriasis 

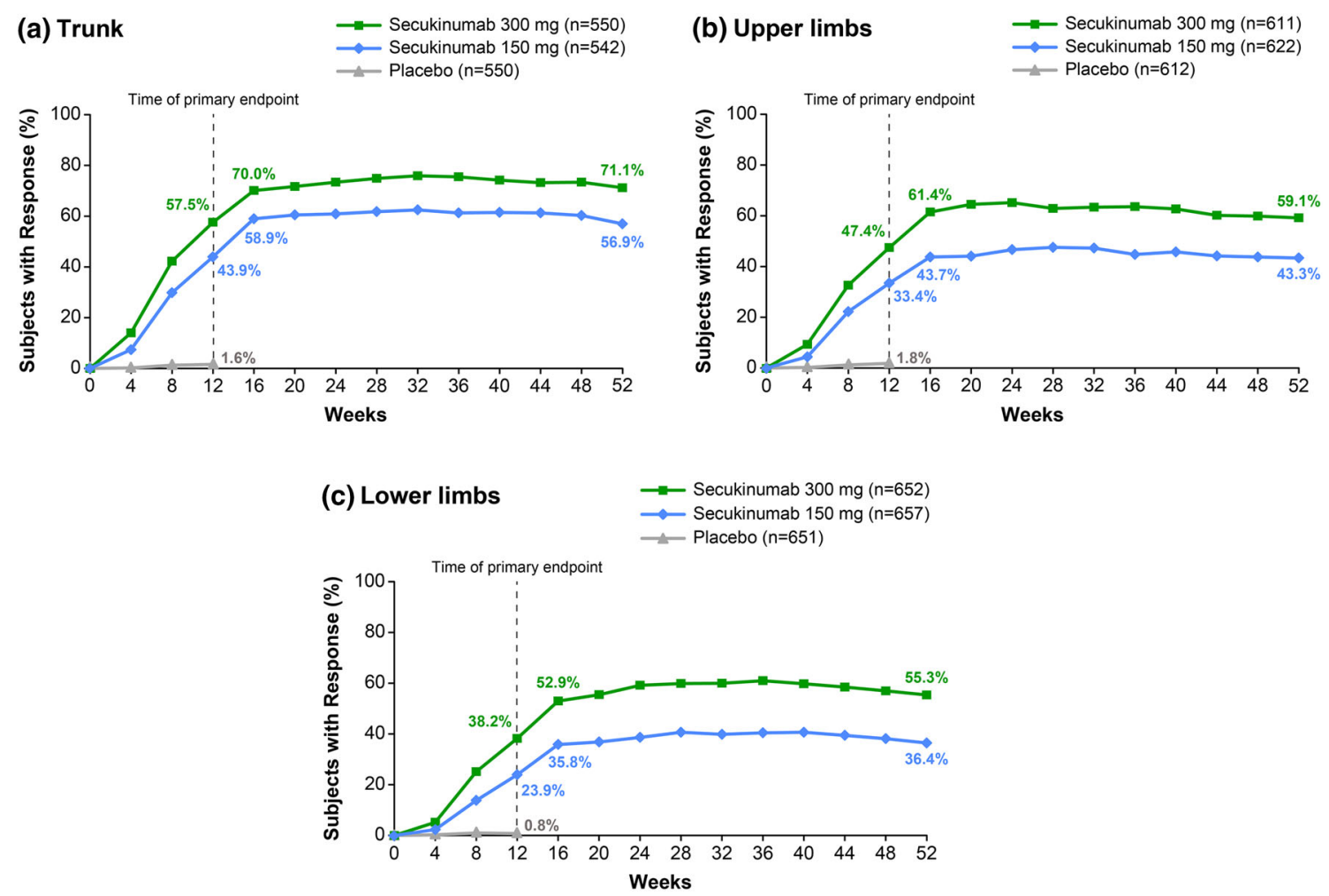

Fig. 2 PASI 100 subscore response rates by body region. Missing values were imputed by multiple imputation. Clinical response rates for a trunk, $\mathbf{b}$ upper limbs, and

regardless of the body region affected. The most robust PASI $90 \backslash 100$ subscore responses at Week 52 with secukinumab $300 \mathrm{mg}$ occurred on the trunk $(78.4 \% \backslash 71.1 \%)$ and the head and neck (76.0\% $\backslash 68.7 \%)$ [8] region. Additionally, a large proportion of patients also achieved PASI $90 \backslash 100$ subscore responses with secukinumab $300 \mathrm{mg}$ on the upper limbs $(67.3 \% \backslash 59.1 \%)$ and the lower limbs (63.9\%\55.3\%) at Week 52. A similar trend was observed with secukinumab $150 \mathrm{mg}$ at Week 52 between the trunk $(66.3 \% \backslash 56.9 \%)$, head and neck $\quad(61.4 \% \backslash 53.1 \%) \quad[8]$, upper limbs $(50.3 \% \backslash 43.3 \%)$, and lower limbs $(45.1 \% \backslash 36.4 \%)$.

More rapid responses were observed with the treatment of secukinumab $300 \mathrm{mg}$ compared with secukinumab $150 \mathrm{mg}$ across all body regions. The time to a 50\% mean reduction in PASI subscore was quickest with c lower limbs from Baseline to Week 52. $n$ Represents the number of evaluable subjects. PASI 100 100\% improvement from Baseline Psoriasis Area and Severity Index

secukinumab $300 \mathrm{mg}$ for the head and neck region (2.0 weeks) [8], followed by the trunk (2.8 weeks), upper limbs (2.9 weeks), and lower limbs (3.4 weeks). A similar trend was reported for secukinumab $150 \mathrm{mg}$, with the time to a $50 \%$ mean reduction in PASI subscore occurring first in the head and neck region (2.7 weeks) [8] followed by the trunk (3.4 weeks), upper limbs (3.7 weeks), and lower limbs (4.5 weeks). Additionally, in ERASURE and FIXTURE, improvement in PASI scores were mirrored by significant improvements in patient-reported symptoms of itching, pain, and scaling compared with placebo up to Week 12 as recorded by the Psoriasis Symptom Diary® [12]. A limitation of this study is the lack of a control group after Week 12. 

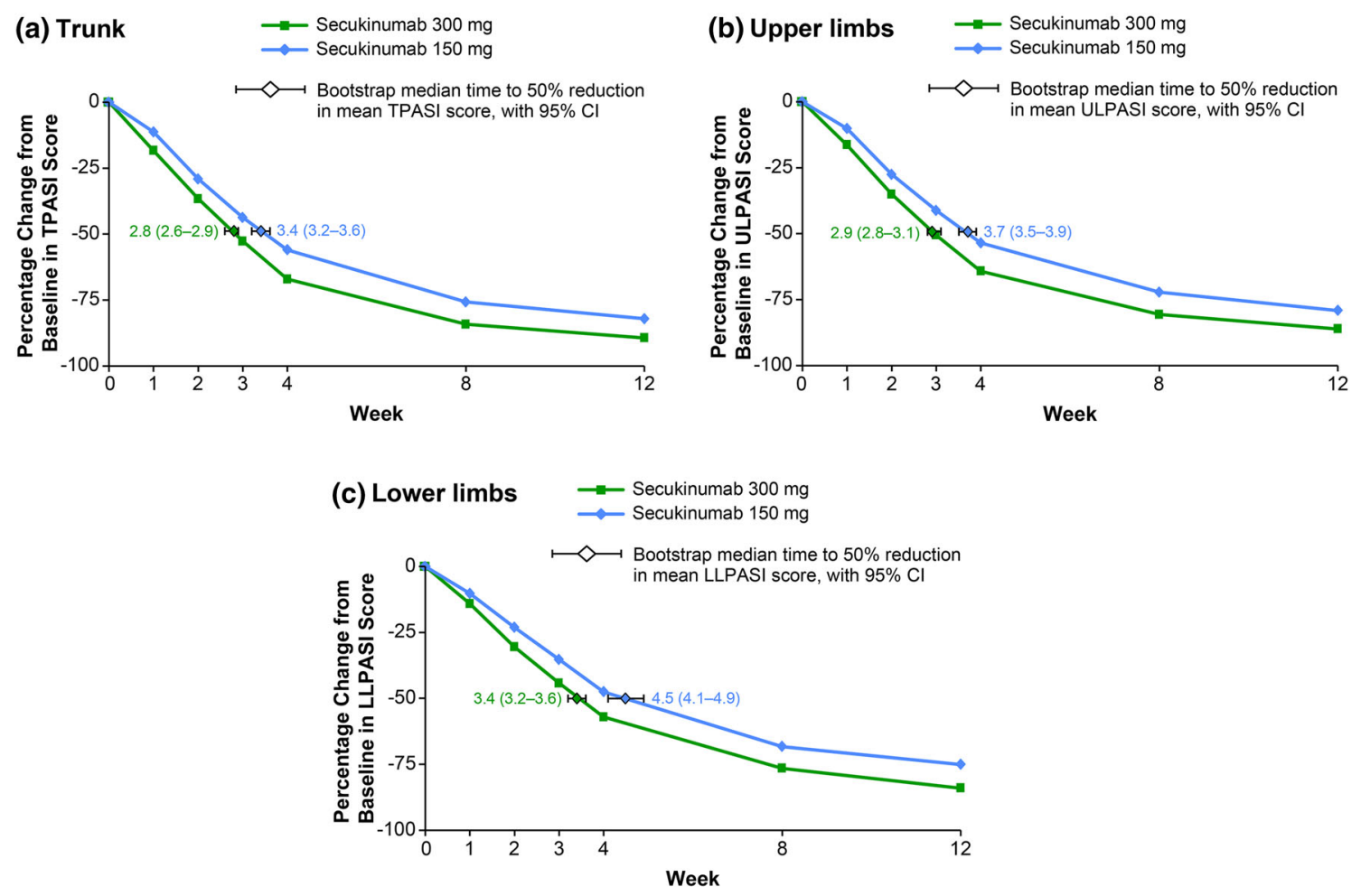

Fig. 3 Percent change in mean PASI subscore over time by body region. A repeated-measures, mixed-effects model was used to analyze the mean percent change from Baseline for a TPASI, $\mathbf{b}$ ULPASI, and c LLPASI subscores. The median time to a $50 \%$ reduction in mean PASI subscore was

Safety findings for the pooled population used in this analysis are reported separately by Kircik et al. [8]. Briefly, adverse events were observed at a similar rate between both secukinumab doses, and the most common adverse events through Week 52 were nasopharyngitis and headache.

Findings from this study are consistent with results from other studies that evaluated treatment efficacy by body region. In these studies, as well as in clinical practice, it has been observed that biologic treatment response typically starts on the head and upper body and progresses downward to lower body regions and extremities [13, 14]. For example, after 16 weeks of treatment with the tumor necrosis factor-alpha inhibitor adalimumab, the greatest estimated from parametric bootstrap samples with the use of linear interpolation between time points. $C I$ confidence interval, LLPASI lower limb Psoriasis Area and Severity Index, TPASI trunk Psoriasis Area and Severity Index, ULPASI upper limb Psoriasis Area and Severity Index

PASI 100 subscore response rates were reported on the head and neck (55.7\%) and the trunk (49.1\%) compared with the upper limbs (33.3\%) and the lower limbs (30.6\%) [14].

\section{CONCLUSION}

In conclusion, the results of our pooled analysis demonstrated that, on the trunk, upper limbs, and lower limbs, secukinumab-especially $300 \mathrm{mg}$-provided PASI 75, PASI 90, and PASI 100 subscore improvements that were rapid and sustained. Disease clearance occurred rapidly on highly visible areas of the skin, which is likely to produce a significant emotional improvement for patients. This study, and the accompanying article by Kircik et al. [8], demonstrate the 
strong efficacy of secukinumab on different body regions. Overall, these results indicate that clear or almost clear skin (PASI 100 or PASI 90) are appropriate treatment goals for patients with moderate-to-severe plaque psoriasis who are treated with secukinumab.

\section{ACKNOWLEDGMENTS}

Previously presented in part at the American Academy of Dermatology (AAD) Annual Meeting, San Francisco, California, United States, 20-24 March 2015. Editorial assistance in the preparation of this manuscript was provided by Oxford PharmaGenesis Inc. Support for this assistance was funded by Novartis Pharmaceuticals Corporation. Sponsorship and article processing charges were funded by Novartis Pharmaceuticals Corporation. All authors had full access to all of the data in this study and take complete responsibility for the integrity of the data and accuracy of the data analysis. All named authors meet the International Committee of Medical Journal Editors (ICMJE) criteria for authorship for this manuscript, take responsibility for the integrity of the work as a whole, and have given final approval to the version to be published. The authors received no financial support or other form of compensation related to the development of this manuscript.

Disclosures. Alan Menter has served as an investigator for Eli-Lilly, Novartis, and Wyeth; has served as an investigator and received research grants from AbbVie, Allergan, Amgen, ApoPharma, Boehringer Ingelheim, Celgene, Convoy Therapeutics, Inc., Genentech, Janssen Biotech, Inc., LEO Pharma, Merck, Pfizer, Symbio \Maruho, and Syntrix; and has served as an advisor, consultant, and $\backslash$ or speaker, and received honoraria from AbbVie, Allergan, Amgen, Boehringer Ingelheim, Convoy Therapeutics, Inc., Eli-Lilly, Genentech, Janssen Biotech, Inc., LEO Pharma, Novartis, Pfizer, Syntrix, Wyeth, and XenoPort.

Jennifer Cather has served as speaker and $\backslash$ or consultant and received honoraria from AbbVie, Celgene, Janssen, LEO Pharma, Medac Pharma, and Novartis; and has served as an investigator and received research grants from Amgen, Celgene, Galderma, Merck, Novartis, Pfizer, Tolmar, Inc., Allergan, GlaxoSmithKline, Sandoz, Vitae, Dermira, Cutanea, Regeneron, and Neothetics.

Michael Jarratt has served as a consultant and received honoraria from Allergan, Galderma, Stiefel Laboratories, Tolmar, Inc., and Valeant; and has served as an investigator and received research grants from Abbott, Allergan, Amgen, Anacor Pharmaceuticals, Inc., Bayer Pharmaceuticals \Intendis, Cutanea Life Sciences, Inc., Galderma, Kythera Biopharmaceuticals, Inc., LEO Pharma A $\backslash$, Lilly Research Laboratories, Lithera, Inc., McNeil PPC, Maruho North America, Inc., Merck \& Co, Merz Pharmaceuticals, LLC, Novartis, Pfizer, Photocure ASA, Stiefel Laboratories, Tolmar, Inc., Topica Pharmaceuticals, Inc., Valeant, and Warner Chilcott.

Xiangyi Meng is an employee and stock holder of Novartis.

Adriana Guana is an employee and stock holder of Novartis.

Judit Nyirady is an employee and stock holder of Novartis.

Compliance with Ethics Guidelines. This article is based on previously conducted studies and does not involve any new studies of human or animal subjects performed by any of the authors. 
Open Access. This article is distributed under the terms of the Creative Commons Attribution-NonCommercial 4.0 International License (http: \\creativecommons.org $\backslash$ licenses $\backslash$ by-nc $\backslash 4.0 \backslash$ ), which permits any noncommercial use, distribution, and reproduction in any medium, provided you give appropriate credit to the original author(s) and the source, provide a link to the Creative Commons license, and indicate if changes were made.

\section{REFERENCES}

1. Langley RG, Elewski BE, Lebwohl M, Reich K, Griffiths CE, Papp $\mathrm{K}$, et al. Secukinumab in plaque psoriasis-results of two phase 3 trials. N Engl J Med. 2014;371:326-38.

2. Blauvelt A, Prinz JC, Gottlieb AB, Kingo K, Sofen H, Ruer-Mulard $\mathrm{M}$, et al. Secukinumab administration by pre-filled syringe: efficacy, safety, and usability results from a randomized controlled trial in psoriasis (FEATURE). Br J Dermatol. 2015;172: 484-93.

3. Paul C, Lacour JP, Tedremets L, Kreutzer K, Jazayeri S, Adams S, et al. Efficacy, safety and usability of secukinumab administration by autoinjector $\backslash$ pen in psoriasis: a randomized, controlled trial (JUNCTURE). J Eur Acad Dermatol Venereol. 2015;29:1082-90.

4. Thaçi D, Blauvelt A, Reich K, Tsai TF, Vanaclocha F, Kingo K, et al. Secukinumab is superior to ustekinumab in clearing skin of subjects with moderate to severe plaque psoriasis: CLEAR, a randomized controlled trial. J Am Acad Dermatol. 2015;73:400-9.

5. Feldman SR, Krueger GG. Psoriasis assessment tools in clinical trials. Ann Rheum Dis. 2005;64(Suppl 2):ii65-8.

6. Augustin M, Reich K, Blome C, Schäfer I, Laass A, Radtke MA. Nail psoriasis in Germany: epidemiology and burden of disease. $\mathrm{Br} \mathrm{J}$ Dermatol. 2010; 163:580-5.
7. Sampogna F, Linder D, Piaserico S, Altomare G, Bortune M, Calzavara-Pinton P, et al. Quality of life assessment of patients with scalp dermatitis using the Italian version of the Scalpdex. Acta Derm Venereol. 2014;94:411-4.

8. Kircik L, Fowler J, Weiss J, Meng X, Guana A, Nyirady J. Efficacy of secukinumab for moderate-to-severe head and neck psoriasis over 52 weeks: pooled analysis of four phase 3 studies. Dermatol Ther (Heidelb). 2016. doi:10.1007\s 13555-016-0139-0. (Online first).

9. Takeshita J, Callis Duffin K, Shin DB, Krueger GG, Robertson AD, Troxel AB, et al. Patient-reported outcomes for psoriasis patients with clear versus almost clear skin in the clinical setting. J Am Acad Dermatol. 2014;71:633-41.

10. Langley RG, Feldman SR, Nyirady J, van de Kerkhof $\mathrm{P}$, Papavassilis C. The 5-point Investigator's Global Assessment (IGA) scale: a modified tool for evaluating plaque psoriasis severity in clinical trials. J Dermatol Treat. 2015;26:23-31.

11. Langley RG, Reich K, Papavassilis C, Fox T, Gong Y, Güttner A. Multiple imputation methodology is reflective of secukinumab efficacy in real clinical practice: data from the FIXTURE and ERASURE studies in moderate-to-severe plaque psoriasis (abstract). Br J Dermatol. 2014;171:e165 (Abstract P199).

12. Strober B, Sigurgeirsson B, Popp G, Sinclair R, Krell J, Stonkus S, et al. Secukinumab improves patient-reported psoriasis symptoms of itching, pain, and scaling: results of two phase 3, randomized, placebo-controlled clinical trials. Int J Dermatol. 2016;55:401-7.

13. Griffiths CE, Sterry W, Brock F, Dilleen M, Stefanidis D, Germain JM, et al. Pattern of response in patients with moderate-to-severe psoriasis treated with etanercept. Br J Dermatol. 2015;172:230-8.

14. Navarini AA, Poulin Y, Menter A, Gu Y, Teixeira HD. Analysis of body regions and components of PASI scores during adalimumab or methotrexate treatment for patients with moderate-to-severe psoriasis. J Drugs Dermatol. 2014;13:554-62. 